\title{
Channel Modeling and Simulation of a Ka-band Videoconferencing System
}

\author{
Reuben A. Farrugia, Carl J. Debono and Paul Micallef \\ Department of Communications \& Computer Engineering \\ University of Malta \\ Msida, Malta \\ rrfarr@eng.um.edu.mt, cjdebo@eng.um.edu.mt, pjmica@eng.um.edu.mt
}

\begin{abstract}
The effect of propagation impairments phenomena is quite significant for systems operating in the Ka-band frequency range, resulting in a reduction in the end-to-end quality of a videoconferencing session. These systems generally utilize complex coding architectures, like the Digital Video Broadcasting (DVB) standard, in order to maintain an acceptable quality of service $(\mathrm{QoS})$ at low signal-to-noise ratios. Although these systems are finding more and more applications, the literature is still relatively poor in the description of models capable of representing such complex architectures. This paper proposes a videoconferencing channel model, developed in software, which can accurately model a complex videoconferencing system according the DVB standard at the required quality and availability.
\end{abstract}

\section{INTRODUCTION}

Bandwidth hungry services, such as videoconferencing applications, transmitted over satellite channels are being pushed at higher frequencies bands where more usable bandwidth is available. However, the effect of propagation impairments is more significant in these frequency bands, and hence the resulting end-to-end quality of the videoconferencing session can be significantly degraded. This effect is even more important under precipitation conditions, where reduction in signal-to-noise (SNR) of about $10 \mathrm{~dB}$ may be observed at low availability.

A number of models were published in the past which can be used to model the propagation impairments in a system operating in Ka-band. Reference [1] lists the models which were found to be most suitable for this videoconferencing system model.

On the other hand, the literature is poor in the analysis of the effects produced by corrupted bits in a compressed video stream. Hardware emulation of the ITALSAT Kaband satellite channel was presented in [2], where the telecommunication systems test-bed of the CNIT National Laboratory for Multimedia Communications has been used. However, such emulators are relatively expensive and are not flexible.

There are a few video system models which are able to model the end-to-end quality of video systems. Richardson [3] has simulated a number of video formats. However, in the analysis [3] assumed a simple two state Markov model, to model the errors in ATM networks, which is not suitable for our system. Max Robert [4] has

This project was funded by the EU $6^{\text {th }}$ Framework Program (FP6) project TWISTER considered the effect of bit errors which occur in bursts on an MPEG2 digital video sequence. Coding architectures with such performance include convolutional, ReedSolomon (RS) and concatenated codes. However, this model is not applicable for systems utilizing Turbo codes.

This paper presents a fast and accurate software simulator of a videoconferencing system which utilizes the Digital Video Broadcasting (DVB) standard, using OPNET Modeler [11]. The model is flexible and can easily be adapted to changing technologies.

The structure of this paper is as follows: Section II provides a general overview of the videoconferencing system considered in this work and gives a brief description of the developed videoconferencing system model. Details of the individual models used by the system model are provided in the following three sections. The simulation results are summarized in Section VI, while the final comments are provided in Section VII.

\section{VIDEOCONFERENCING SYSTEM}

Eutelsat's Hotbird 6, which is a geostationary (GEO) satellite, located at $13^{\circ}$ East, is used to deliver point-topoint multimedia content between the University of Malta and its branch in the sister island of Gozo. The videoconference camera is used to capture the live videoconferencing session, and encodes the video, audio and data according the H.323 protocol [5]. This data is encapsulated within IP datagrams and passed to the SKYPLEX module, which encodes and modulates the datagrams according to the DVB standard.

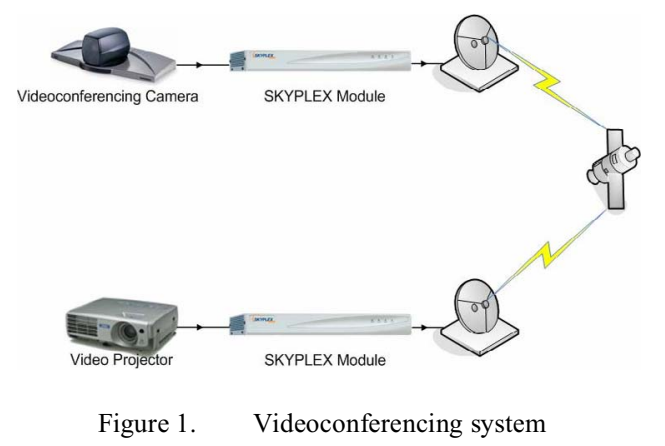

The videoconferencing system operates in the Ka-band at an uplink frequency of $29.5 \mathrm{GHz}$ and a downlink frequency of $19.7 \mathrm{GHz}$ and utilizes dual linear polarization at an elevation angle of $48.71^{\circ}$. The coverage of the GEO satellite with respect to both earth stations is 
about $5 \mathrm{~dB}$ less than its peak value. Therefore, this system is more susceptible to degradation in both performance and quality.

The channel model of this system can be divided into three independent sub-models: (1) generation of the packets produced during a real videoconferencing session, (2) modeling the performance of the forward error correction (FEC) codes used by the SKYPLEX module for both the uplink and the downlink channels, and (3) modeling of the propagation impairments effecting both the uplink and the downlink satellite communication links. Fig. 2 illustrates the videoconferencing system model considered in this work.

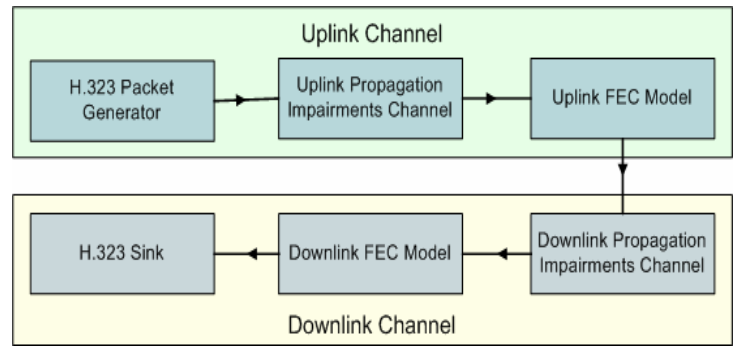

Figure 2. Videoconferencing system model

\section{IP PACKET GENERATOR MODEL}

The H.323 protocol is an umbrella protocol which encodes real-time video, audio and data communication over the IP packet-switched network. The H.323 terminal compresses both audio and video streams, so that they can be efficiently delivered. Unreliable User Datagram Protocol (UDP) end-to-end services are used for the transmission of both audio and video information. The resulting H.323 packets are encapsulated within IP datagrams and passed to the SKYPLEX module before being transmitted to the remote base station.

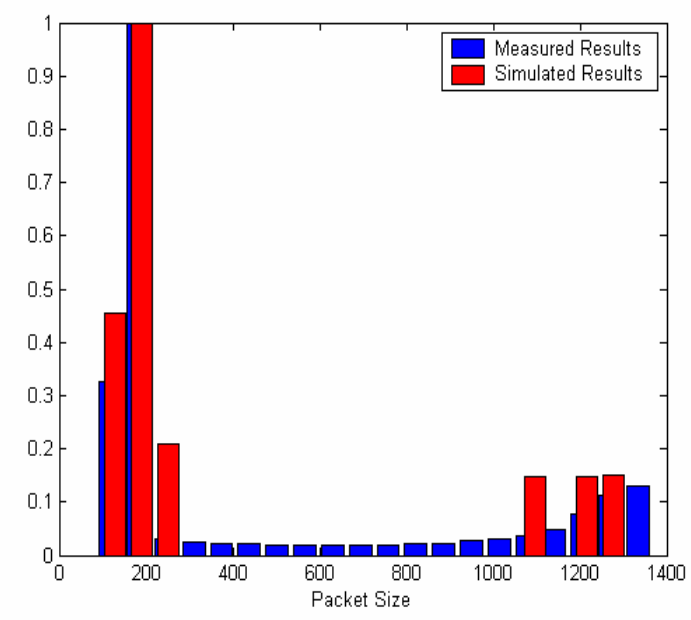

Figure 3. Normalized pakect size histogram

Live videoconferencing sessions were captured and both arrival time and packet size were logged. After analyzing the data, it was found that the distribution of the inter-arrival time can be approximated by an exponential distribution. On the other hand, the packet size distribution did not closely match any standard probability density function (PDF) as shown in Fig. 3. A non-standard PDF based on the normalized histogram of the collected data was therefore generated.

\section{FEC PERFORMANCE MODELING}

The received IP datagrams are fragmented and encapsulated within MPEG2 TS frames [6], 188 bytes each. The SKYPLEX module utilizes FEC codes on both uplink and downlink channels in order to protect these frames, thus making the videoconferencing quality acceptable even at low SNR.

The FEC codes were simulated using Mote Carlo methods [7] with a level of confidence of $95 \%$ and a tolerance of $10 \%$. In both cases, an additive white Gaussian noise (AWGN) channel was assumed. The data collected during these simulations was used to develop fast software emulators, which can derive the performance of the coding scheme with negligible loss in accuracy.

\section{A. Uplink Channel Simulator}

The SKYPLEX module utilizes turbo codes in the uplink channel according to the DVB-RCS standard [8]. The turbo encoder utilizes duo-binary Circular Recursive Systematic Convolutional (CRSC) in conjunction with the tail-biting technique which allows the encoded data to be represented by a circular trellis, without reducing the code rate. Using double-binary codes as component codes reduce the effect of puncturing.

The decoder utilizes a modified version of the BCJR algorithm [9] which is computed in the logarithmic domain. This makes the Maximum a Posteriori (MAP) decoder less computational intensive and less sensitive to rounding-off errors. Fig. 4 illustrates the expected performance of the DVB-RCS forward error correction code.

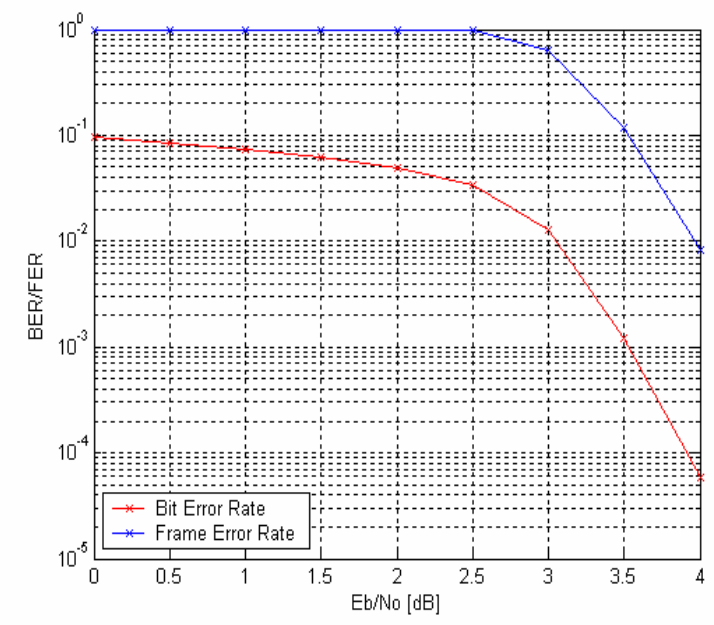

Figure 4. Uplink FEC performance

\section{B. Downlink Channel Simulator}

The GEO satellite encodes on-board the received MPEG2 TS frames according to the DVB-S standard [10]. 
This standard is based on outer Reed-Solomon codes, inner convolutional codes and an intermediate convolutional interleaver.

The outer Reed-Solomon $(204,188)$ shortened code is capable of correcting 8 corrupted bytes. The convolutional interleaver of depth 12 is placed between the outer and inner codes. The inner convolutional code has code rate of $1 / 2$ with a constraint length of 7 . A code rate of $3 / 4$ is achieved by applying the puncturing technique. Fig. 5 illustrates the expected performance downlink FEC code.

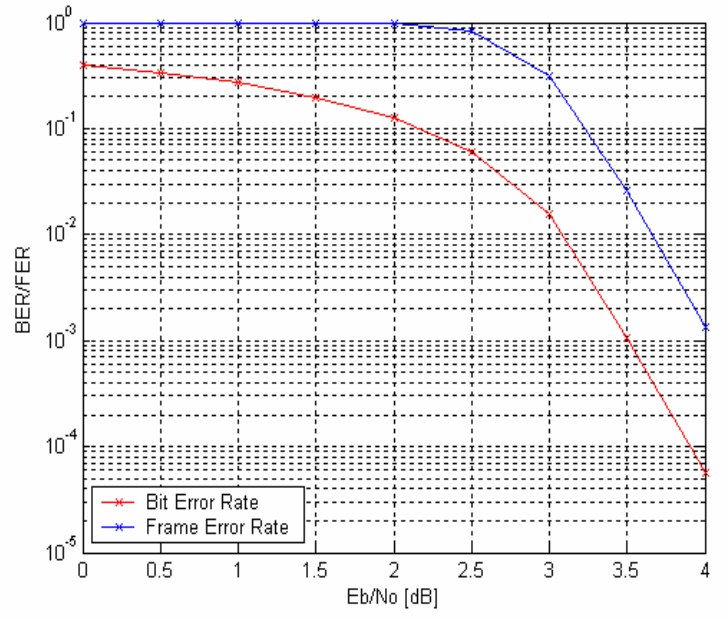

Figure 5. Downlink FEC performance

\section{PROPAGation IMPAirments MOdeling}

The quality of service (QoS) of videoconferencing systems operating in the Ka-band is significantly degraded because of the propagation impairments present in the earth-satellite links. These propagation impairments include attenuation phenomena such as gaseous absorption, cloud and rain attenuation, and tropospheric scintillation. Moreover, for systems utilizing frequency reuse with orthogonal polarization, cross polar interference should be considered.

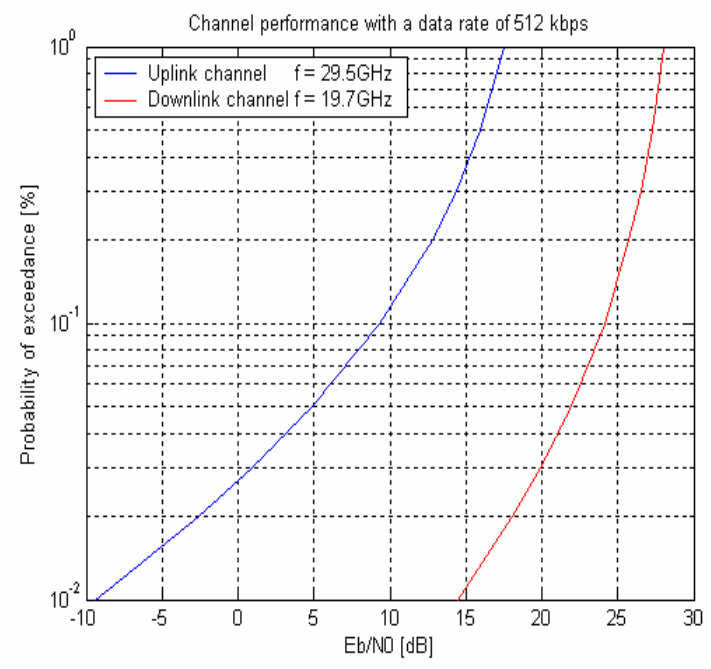

Figure 6. Uplink and Downlink channel performance
The effect of the propagation impairments is subject to uncertainty because of the unpredictability of the local weather. Long term prediction models which model the attenuation, noise and interference affecting a system operating in the Ka-band were described in [1]. These models make use of meteorological information to model such propagation impairments at the required system availability. The uplink and downlink channel performance for the videoconferencing system operating at a data rate of $512 \mathrm{kbps}$ is shown in Fig. 6.

\section{Simulation Results}

The videoconferencing system was modeled on OPNET Modeler [11], which is the industry's leading environment for network modeling and simulation. Fig. 7 illustrates the network level of the considered videoconferencing simulation. Each sub-network contains nodes which provide lower level modeling of the required system. Both propagation impairments and forward error correction models were developed and integrated within the Radio Transceiver Pipeline procedures available within OPNET Modeler [11]. The videoconferencing traffic generator was modeled within OPNET's process model and was used to generate the traffic present during a videoconferencing session using the distributions mentioned in Section III.

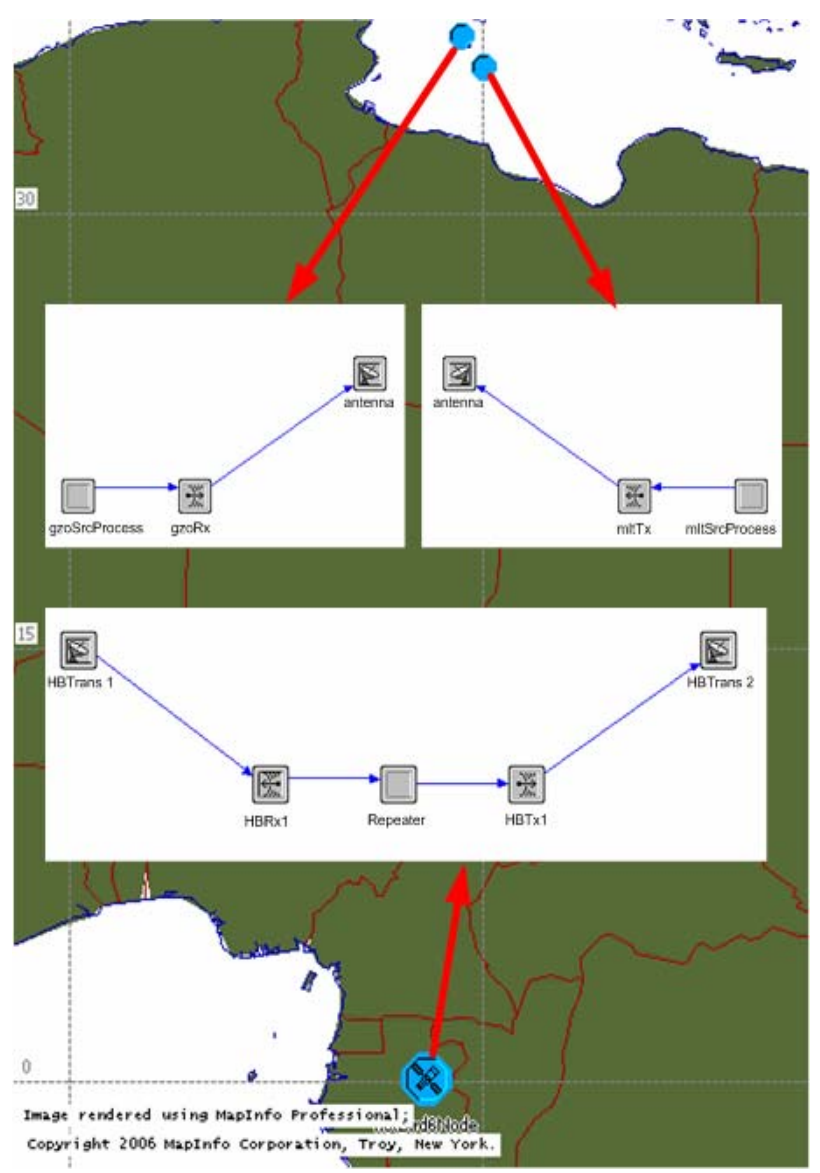

Figure 7. OPNET Modeler simulation of a videoconferencing system between the University of Malta and its branch in Gozo 
The videoconferencing traffic generator data was first compared to the experimental data captured during the live videoconferencing sessions to confirm its validity. The results obtained are summarized in Table I, where a data rate of $512 \mathrm{kbps}$ was used in both cases. The correlation between the simulated and measured results is evident. The discrepancies between the simulation and measured results are mainly present because of the approximated non-standard packet size distribution.

TABLE I. SIMULATION RESULTS

\begin{tabular}{|l|c|c|}
\hline \multicolumn{3}{|c|}{ Network parameters } \\
\hline \multicolumn{1}{|c|}{ Characteristics } & Simulated & Measured \\
\hline Throughput Packets / Sec & 104.06 & 107.912 \\
\hline Throughput Bits / Sec & 330919 & 403949 \\
\hline Average Packet Size (bytes) & 397.5 & 467.913 \\
\hline Mean Arrival Time & 0.0092 & 0.0093 \\
\hline
\end{tabular}

The videoconferencing simulator illustrated in Fig. 7 was used to derive the bit-error-rate (BER) and frameerror-rate (FER) of the videoconferencing system at different probability of exceedance. Due to the powerful FEC codes used in both the uplink and the downlink channel, the quality of the videoconference session is expected to be poor at low probability of exceedance values. In fact, the system is expected to operate in the quasi error free connection $\left(\mathrm{BER}=10^{-9}-10^{-10}\right.$ ) for probability of exceedance superior to $0.05 \%$. Fig. 8 illustrates the expected BER and FER of the videoconference system at low probability of exceedance values. These results confirm that Ka-Band videoconferencing systems provide a solution for providing content to remote rural areas, especially in regions where the probability of exceedance is low.

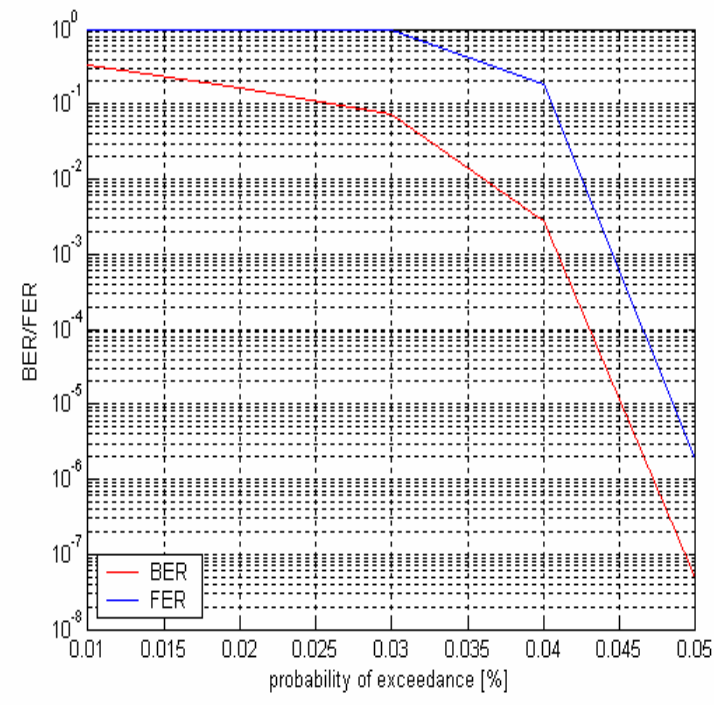

Figure 8. BER and FER simulated performance

\section{COMMENTS AND CONCLUSION}

The end-to-end quality of a number of live videoconferencing sessions between the University of Malta and its branch in Gozo were characterized using the method described in [1]. The resulting BER and FER were found to be zero, since generally the system is operating in a quasi error free connection. However, under heavy precipitation the channel will become lossy and a number of packets will be lost, resulting in a significant degradation of the end-to-end quality of the system.

Objective evaluation of the video quality will be implemented in the future. Moreover, since the H.323 terminal utilizes channel coding to achieve efficient transmission of the video and audio streams, the quality of the videoconferencing session is not only related to first order quality metrics such as the BER and FER. Higher order modeling of the FEC models should be used so that the actual location of erroneous bits within a frame is derived.

\section{ACKNOWLEDGMENT}

This project was funded by the EU $6^{\text {th }}$ Framework Program (FP6) project TWISTER. The authors would like to thank EUTELSAT, in particular Dr Hector Fenech and Dr Emmanuel Lance for the provided information.

\section{REFERENCES}

[1] R. A. Farrugia, C. J. Debono and P. Micallef, "Propagation Impairments Modeling and QoS Parameters Characterization in a Ka-band Videoconferencing system", Proc. of EUROCON 2005 - The International Conference on "Computer as a Tool", pp. 453456, Nov. 2005.

[2] N. Celandroni, F. Davoli, E. Ferro, S. Vignolo, S. Zappatore, and A. Zinicola, "An Experimental Study on the Quality-of-Service of Video Encoded Sequences Over an Emulated Rain-Faded Satellite Channel", IEEE Journal on Selected Areas in Communications, vol. 22, No. 2, pp. 229-237, Feb. 2004

[3] I. E. G. Richardson, "Video Coding for Reliable Communications", Ph.D dissertation, Robert Gordon University, Oct. 1999.

[4] P. M. Robert, A. M. Darwish and J. H. Reed, "MPEG video quality prediction in a wireless system", Proc. of the IEEE $49^{\text {th }}$ Vehicular Technonogy Conference, vol. 2, pp. 1490-1495, Jul. 1999.

[5] ITU-T Recommendation H.323, "Infrastructure of audiovisual services - Systems and terminal equipment for audiovisual services”, Series H: Audiovisual and Multimedia Systems, 2003.

[6] ETSI EN 301 192, "Digital Video Broadcasting (DVB); DVB specification for data broadcasting", 2004.

[7] M. C. Jeruchim, P. Balaban and K. S. Shanmugan, "Simulation of Communication Systems - Second Edition", Plenum Press, New York, 2000.

[8] ETSI EN 301 790, "Digital Video Broadcasting (DVB); Interactive channel for satellite distribution systems", 2005.

[9] L. R. Bahl, J. Cocke, F. Jelinek and J. Raviv, "Optimal Decoding of Linear Codes for Minimizing Symbol Error Rate", IEEE Transactions on Information Theory, pp. 284-287, Mar. 1974.

[10] ETSI EN 300 421, "Digital Video Broadcasting (DVBFraming structure, channel coding and modulation for $11 / 12 \mathrm{GHz}$ satellite services", 1997.

[11] OPNET Inc., "Opnet Modeler: The World's Leading Network Modeling and Simulation Environment," [Online document], May 2004, available at www.opnet.com/products/modeler/home.html. 